\title{
In vivo analysis of insulin-like growth factor type 1 receptor humanized monoclonal antibody MK-0646 and small molecule kinase inhibitor OSI-906 in colorectal cancer
}

\author{
PREMILA D. LEIPHRAKPAM, EKTA AGARWAL, MICHELLE MATHIESEN, \\ KATIE L. HAFERBIER, MICHAEL G. BRATTAIN and SANJIB CHOWDHURY \\ Eppley Cancer Center, University of Nebraska Medical Center, Omaha, NE 68198-5950, USA
}

Received August 16, 2013; Accepted September 20, 2013

DOI: $10.3892 / o r .2013 .2819$

\begin{abstract}
The development and characterization of effective anticancer drugs against colorectal cancer (CRC) is of urgent need since it is the second most common cause of cancer death. The study was designed to evaluate the effects of two IGF-1R antagonists, MK-0646, a recombinant fully humanized monoclonal antibody and OSI-906, a small molecule tyrosine kinase inhibitor on CRC cells. Xenograft study was performed on IGF-1R-dependent CRC cell lines for analyzing the antitumor activity of MK-0646 and OSI-906. Tumor proliferation and apoptosis were assessed using Ki67 and TUNEL assays, respectively. We also performed in vitro characterization of MK-0646 and OSI-906 treatment on CRC cells to identify mechanisms associated with drug-induced cell death. Exposure of the GEO and CBS tumor xenografts to MK-0646 or OSI-906 led to a decrease in tumor growth. TUNEL analysis showed an increase of approximately $45-55 \%$ in apoptotic cells in both MK-0646 and OSI-906 treated tumor samples. We report the novel finding that treatment with IGF-1R antagonists led to downregulation of X-linked inhibitor of apoptosis (XIAP) protein involved in cell survival and inhibition of cell death. In conclusion, IGF-1R antagonists (MK-0646 and OSI-906) demonstrated single agent inhibition of subcutaneous CRC xenograft growth. This was coupled to pro-apoptotic effects resulting in downregulation of XIAP and inhibition of cell survival. We report a novel mechanism by which MK-0646 and OSI-906 elicits cell death in vivo and in vitro. Moreover, these results indicate that MK-0646 and OSI-906 may be potential anticancer candidates for the treatment of patients with IGF-1R-dependent CRC.
\end{abstract}

Correspondence to: Dr MichaelG.Brattain or DrSanjib Chowdhury, Eppley Cancer Center, University of Nebraska Medical Center, 985950 Nebraska Medical Center, Omaha, NE 68198-5950, USA

E-mail: mbrattain@unmc.edu

E-mail: schowdhury@unmc.edu

Key words: colorectal cancer, IGF-1R, MK-0646, OSI-906, antitumor activity, X-linked inhibitor of apoptosis, cell survival

\section{Introduction}

The insulin-like growth factor receptor (IGF-1R) is a receptor tyrosine kinase that is widely expressed in normal human tissues and upregulated in a number of human cancers including colorectal cancer (CRC) (1-3). IGF-1R is comprised of three components, two extracellular $\alpha$-chains, that are involved in ligand binding, two transmembrane spanning $\beta$-chains and an intracellular tyrosine kinase $(2,4,5)$. Both IGF1 and IGF2 are ligands for IGF-1R and their binding induces receptor autophosphorylation at the tyrosine kinase domain, resulting in its activation by a conformational change leading to stimulation of signaling cascades, including PI3K/Akt and MAPK pathways (4-8). Activation of IGF-1R has been reported to stimulate oncogenic cellular processes, including aberrant cell survival mechanisms, transformation, motility, angiogenesis and metastasis $(2,3,9)$. Previous work from our laboratory as well as other groups has shown that inhibition of IGF-1R has been shown to impede tumorigenesis in several human xenograft models $(2,3,9,10)$.

IGF-IR plays a multifunctional role in human CRC growth and is widely regarded as an attractive target for anticancer drug treatment based on the observation that inhibition of IGF-1R function results in apoptosis and inhibition of tumor growth. Several pharmacological strategies are currently being adopted in clinical trials to disrupt the IGF-IF signaling pathway. This includes anti-receptor antibodies to reduce receptor expression, small-molecule IGF-1R kinase inhibitors, and targeting downstream IGF-1R signaling pathways with agents, such as Akt or mTOR inhibitors (9). In this study, we characterized the in vivo and in vitro effects of MK-0646, a novel IGF-1R recombinant humanized monoclonal antibody. It has been reported that MK-0646 binds to IGF-1R and triggers receptor internalization and degradation thereby blocking IGF-1 and II mediated cellular proliferation and survival (11). MK-0646 specifically targets IGF-1R and does not cross-react with the insulin receptor (12). It is in phase II clinical trial at present (13-16).

OSI-906 is a potent and highly selective small molecule tyrosine kinase inhibitor which binds dually to IGF-1R and IR and inhibits autophosphorylation (6,7). It is also in phase II clinical trials at present (16). Initiation of apoptosis and inhibition of cell proliferation following OSI-906 treatment appears 
to be directly linked to Akt inhibition in various tumor cell lines including lung, pancreatic and CRC cell lines $(6,17)$. In addition, OSI-906 has shown potent antitumor activity in vivo in several xenograft models (18). Buck et al has shown that OSI-906 reduces tumorigenicity in GEO CRC xenografts (18). However, the signaling mechanisms associated with OSI-906mediated cell death are poorly understood.

The goal of the present study was to compare the antagonistic effects of MK-0646 and OSI-906 in vivo and in vitro and characterize mechanisms associated with drug-induced cell death. We report for the first time the antitumor activity of MK-0646 in IGF-1R-dependent CRC cells and demonstrate that inhibition of IGF-1R leads to control of aberrant cell survival signaling through the downregulation of XIAP and induction of cell death.

\section{Materials and methods}

Cell lines. GEO and CBS cell lines used in this study were originally developed from primary $\mathrm{CRC}$ tumors and have been extensively characterized (19). Cells were maintained at $37^{\circ} \mathrm{C}$ in humidified atmosphere of $5 \% \mathrm{CO}_{2}$ in a chemically defined serum-free medium consisting of McCoy's 5A medium (Sigma-Aldrich, St. Louis, MO, USA) supplemented with amino acids, pyruvate, vitamins, antibiotics and growth factors transferring (4 $\mu \mathrm{g} / \mathrm{ml}$; Sigma-Aldrich), insulin $(20 \mu \mathrm{g} / \mathrm{ml}$; Sigma-Aldrich), and EGF (10 ng/ml; R\&D Systems) as previously described (20). Supplemented McCoy's medium ('SM') is McCoy's 5A medium supplemented with antibiotics and nutrients but lacking any growth factors. Cells were routinely subcultured with a $0.25 \%$ trypsin (Invitrogen, Carlsbad, CA, USA) in Joklik's medium (Invitrogen) containing 0.1\% EDTA. When cells were under growth factor deprivation status (GFDS), they were cultured in SM medium without growth factor or serum supplements for the indicated time periods without medium change in between.

Antibodies. IGF-1R $\beta$, pIGF-1R $\beta\left(\mathrm{Y}^{1135}\right)$ and p21 antibodies were obtained from Cell Signaling Technology Inc. (Beverly, MA, USA). XIAP antibody was obtained from abcam. $\beta$-actin and GAPDH antibodies were from Sigma-Aldrich (St. Louis, MO, USA).

Pharmacological antagonists. MK-0646 was provided by Merck \& Co. (Whitehouse Station, NJ, USA) and OSI-906 was purchased from Chemitek, Indianapolis, IN, USA.

Xenograft experiments. All experiments involving animals were approved by the University of Nebraska Medical Center Institutional Animal Care and Use Committee. The GEO and CBS cells were transfected with green fluorescence protein (GFP). Exponentially growing GFP-labeled GEO and CBS cells $(\sim 7$ million cells $/ \mathrm{ml}$ SF media) were inoculated subcutaneously onto the dorsal surfaces of athymic nude male mice and the growth of the tumor was monitored by biweekly measurements using a caliper. Once xenografts were established ( $\left.\sim 50-100 \mathrm{~mm}^{3}\right)$, MK-0646 or OSI-906 treatment was initiated and continued for two weeks. MK-0646 was given by intraperitoneal (IP) injection weekly ( $20 \mathrm{mg} / \mathrm{kg}$ ) on both GEO and CBS xenografted mice for three doses and formulation buffer was the vehicle. OSI-906 was given by daily oral gavage (40 mg/kg) on GEO xenografted mice and tartaric acid was the vehicle. Xenografts were harvested after 14 days of treatment for assessment of molecular effects by the two agents.

Xenograft lysate preparation. Xenografts were harvested and snap frozen in liquid nitrogen and stored at $-80^{\circ} \mathrm{C}$. Xenografts were first washed in cold 5\% PBS and collected in lysis buffer [50 mmol/1 Tris (pH 7.4), $100 \mathrm{mmol} / 1 \mathrm{NaCl}, 1 \%$ NP40, $2 \mathrm{mmol} / \mathrm{l}$ EDTA, 0.1\% SDS, $50 \mathrm{mmol} / 1 \mathrm{NaF}, 10 \mathrm{mmol} / \mathrm{l}$ $\mathrm{Na}_{3} \mathrm{VO}_{4}, 1 \mathrm{mmol} / 1$ phenylmethylsulfonyl fluoride, $25 \mu \mathrm{g} / \mathrm{ml}$ $\beta$-glycerophosphate, and one protease inhibitor cocktail tablet from Roche]. Crude xenograft lysates were homogenized to shear DNA and lysed for $30 \mathrm{~min}$ on ice. Xenograft lysates were then cleared by centrifugation at $13000 \mathrm{rpm}$ for $20 \mathrm{~min}$ at $4^{\circ} \mathrm{C}$. Protein concentrations were determined by the Pierce bicinchonimic acid protein assay (Pierce Biotechnology, Inc., Rockford, IL, USA).

Cell lysate preparation. GEO CRC cells were allowed to grow until 70-80\% confluent in 100-mm culture plates and were treated with different concentrations of either MK-0646 or OSI-906 under growth factor deprivation status (GFDS) for $48 \mathrm{~h}$. Cells were washed in cold 5\% PBS and collected in lysis buffer. Crude cell lysates were homogenized using a 21-gauge needle to shear DNA and lysed for $30 \mathrm{~min}$ on ice. Cell lysates were then cleared by centrifugation at $13000 \mathrm{rpm}$ for $20 \mathrm{~min}$ at $4^{\circ} \mathrm{C}$. Protein concentrations were determined by the Pierce bicinchonimic acid protein assay (Pierce Biotechnology, Inc.).

Western blot analysis. Protein (30-100 $\mu \mathrm{g})$ was fractionated on an acrylamide denaturing gel and transferred onto a nitrocellulose membrane (Amersham Biosciences) by electroblotting. The membrane was blocked with $5 \%$ nonfat dry milk in $1 \mathrm{X}$ TBST (50 mM Tris, pH 7.5, $150 \mathrm{mM} \mathrm{NaCl,} \mathrm{0.05 \%} \mathrm{Tween-20)}$ for $1 \mathrm{~h}$ at room temperature or overnight at $4^{\circ} \mathrm{C}$. The membrane was then incubated with primary antibodies for $1 \mathrm{~h}$ at room temperature or overnight at $4{ }^{\circ} \mathrm{C}$ with $5 \%$ nonfat dry milk in $1 \mathrm{X}$ TBST or $5 \%$ bovine serum albumin (BSA) in $1 \mathrm{X}$ TBST according to the manufacturer's instructions. After washing three times with $1 \mathrm{X}$ TBST for $10 \mathrm{~min}$ each, the membrane was incubated with horseradish peroxidase-conjugated secondary antibody (Amersham Biosciences) for $1 \mathrm{~h}$ at room temperature. After further washing in $1 \mathrm{X}$ TBST three times for $10 \mathrm{~min}$ each, the proteins were detected by the enhanced chemiluminescence system (Amersham Biosciences).

Cell death assays. DNA fragmentation assays were performed on cells treated with either MK-0646 or OSI-906 under GFDS. Cells were seeded in 96-well plates and allowed to grow to $70-80 \%$ confluence. The cells were then changed to Supplemented McCoy's medium ('SM') and treated with various concentrations of either MK-0646 or OSI-906 for $48 \mathrm{~h}$. Assays were then performed using a cell death ELISA kit (Roche Applied Science) according to the manufacturer's protocol as previously described (21). The plate was read at $405 \mathrm{~nm}$. Inhibition of proliferation was assessed by the MTT [3-(4,5-dimethylthiazol-2-yl)-2,5-diphenyltetrazolium bromide] assay as previously described (22). 

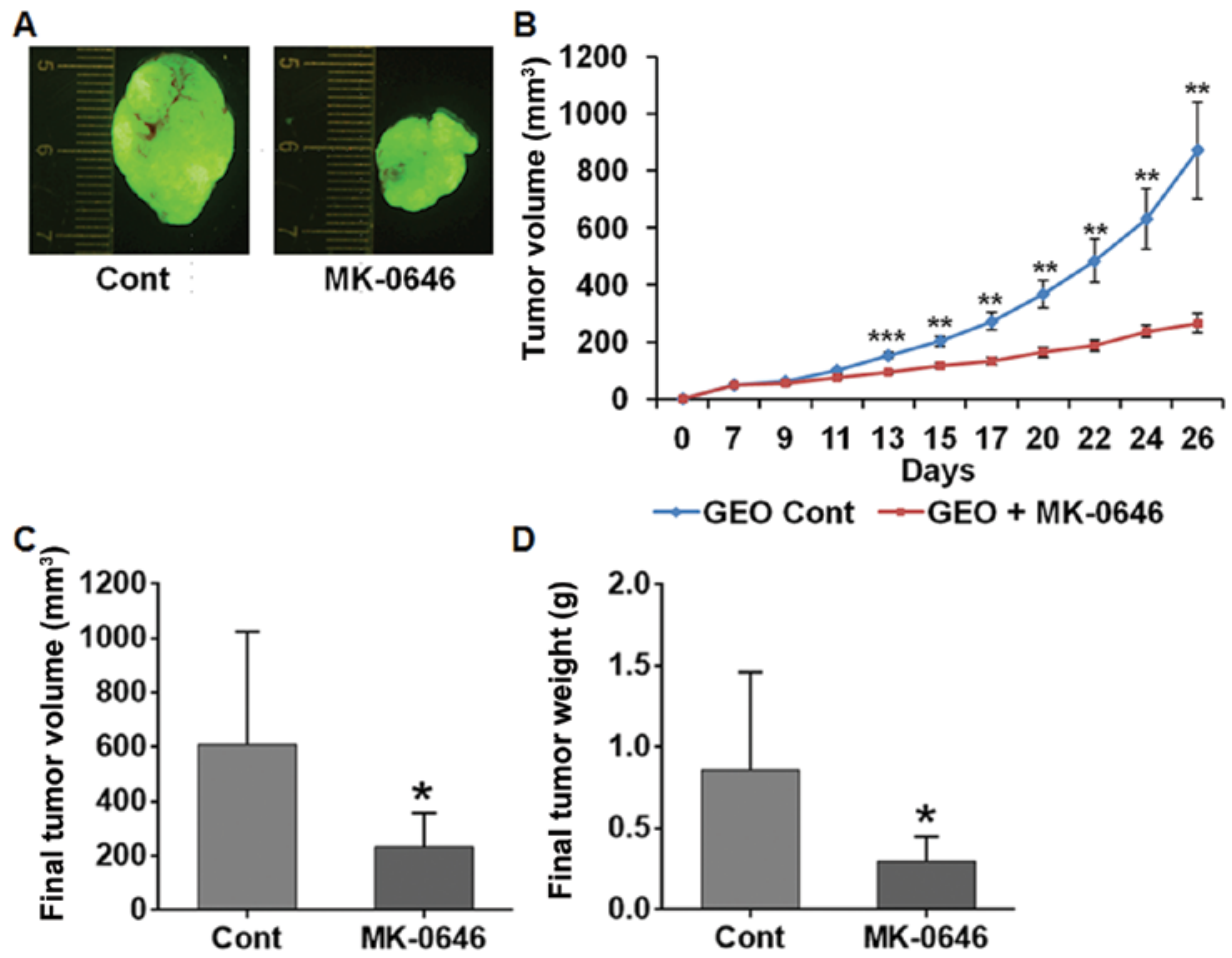

Figure 1. In vivo analysis of IGF-1R inhibition using MK-0646 on tumor growth and weight in GEO CRC xenografts. Significant decrease of the tumor volume and weight was observed in MK-0646 treated xenografts compared with the control (A, B, C and D). (n=14, control=7, MK-0646 treated=7).

Cell cycle arrest analysis. GEO CRC cells were allowed to grow until $70-80 \%$ confluent in 100 -mm culture plates and were treated with different concentrations of either MK-0646 or OSI-906 under growth factor deprivation status (GFDS) for $48 \mathrm{~h}$ and cell cycle analysis was performed as previously described (23).

Hematoxylin and eosin, TUNEL and Ki67 staining. Xenografts obtained from subcutaneous injection of GEO cells were harvested and placed in $10 \%$ neutral buffer formalin fixative for 12-24 $\mathrm{h}$ and then embedded in paraffin. Sections $(4 \mu \mathrm{m})$ were cut from paraffin-embedded blocks using a microtome and were used for hematoxylin and eosin stains and immunohistochemical characterizations. Serial sections were cut to complement the hematoxylin and eosin sections and were stained with the Apo-tag (Millipore) terminal nucleotidyl transferase mediated nick end-labeling (TUNEL) kit following the manufacturer's protocol. The apoptotic rate was determined semi-quantitatively by counting the number of positively stained apoptotic bodies per $75-\mu \mathrm{m}^{2}$ field at a magnification of $x 20$. Approximately 1000 total cells were counted and the percentages of positively stained cells were calculated. Four control and four treated slides for MK-0646 and OSI-906 were analyzed, respectively. Staining was also performed with $\operatorname{IgG}_{1}$ rabbit polyclonal antibody for Ki67 (Dako Corp.). Ki67 is a non-histone nuclear antigen present in late $\mathrm{G}_{1}, \mathrm{G}_{2}$, and $S$ phases of the cell cycle but not in $\mathrm{G}_{0}$. A 1:20 dilution was used and staining was performed following the manufacturer's protocol. The proliferation rate was determined semi-quantitatively by counting the number of positively stained proliferative cells per $75-\mu \mathrm{m}^{2}$ field at a magnification of $\mathrm{x} 20$. Approximately 1000 total cells were counted and the percentages of positively stained cells were calculated. Four control and four treated slides for MK-0646 and OSI-906 were analyzed, respectively.

Immunohistochemistry. Sections $(4 \mu \mathrm{m})$ were cut from the paraffin-embedded xenograft tumor blocks, deparaffinized in histoclear, and rehydrated in descending grades of ethanol. Endogenous peroxidase activity was blocked with $3 \%$ hydrogen peroxide in water. Immunostaining was performed for XIAP using an indirect detection method (24). The staining was accompanied by a negative control in which slides were incubated with a matching blocking peptide to the primary antibody. Slides were counterstained with hematoxylin. Specimens were processed on the same day to eliminate any variability in conditions. Slides were digitally photographed using the same settings.

Statistical analysis. Statistical significance was determined using two-tailed Student's t-test with a p-value $<0.05$. All the experiments were repeated three times independently to determine consistency in the results. The results were expressed as mean $\pm \mathrm{SE}$ for three replicates for each treatment.

\section{Results}

Inhibition of IGF-1R is associated with antitumor activity in vivo. The antitumor effects were determined in the IGF-1Rdependent CRC sub-cutaneous xenograft tumors following treatment with IGF-1R antagonist MK-0646 or OSI-906. MK-0646 treatment for three weeks at $20 \mathrm{mg} / \mathrm{kg}$ dose once weekly inhibited the growth of the GEO xenograft tumors (Fig. 1A and B). Similar results were obtained in MK-0646 treated CBS xenograft tumor (data not shown). The final tumor 
A

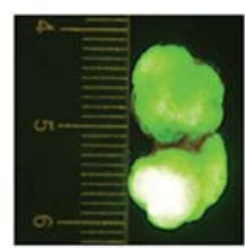

Cont

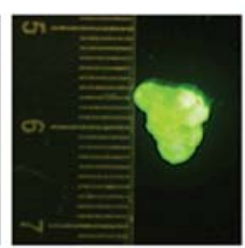

OSI-906
B

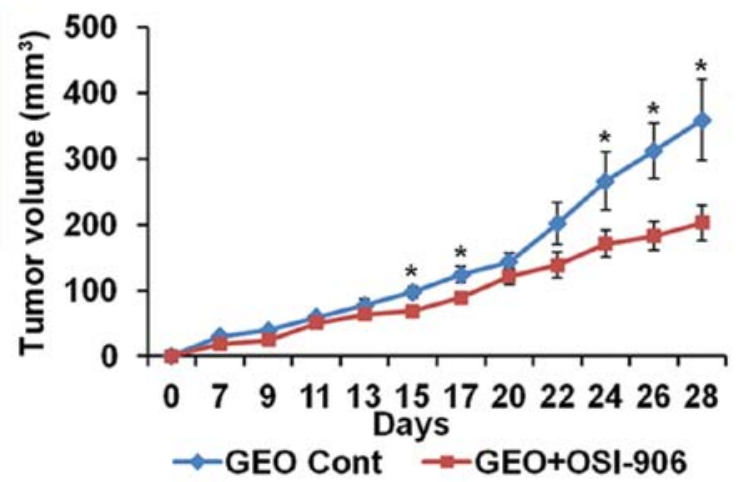

Figure 2. In vivo analysis of IGF-1R inhibition using OSI-906 on tumor growth and weight in GEO CRC xenografts. Significant decrease of the tumor volume was observed in OSI-906 treated xenografts compared with the control (A and B). ( $\mathrm{n}=9$, control=4, OSI-906 treated=5).
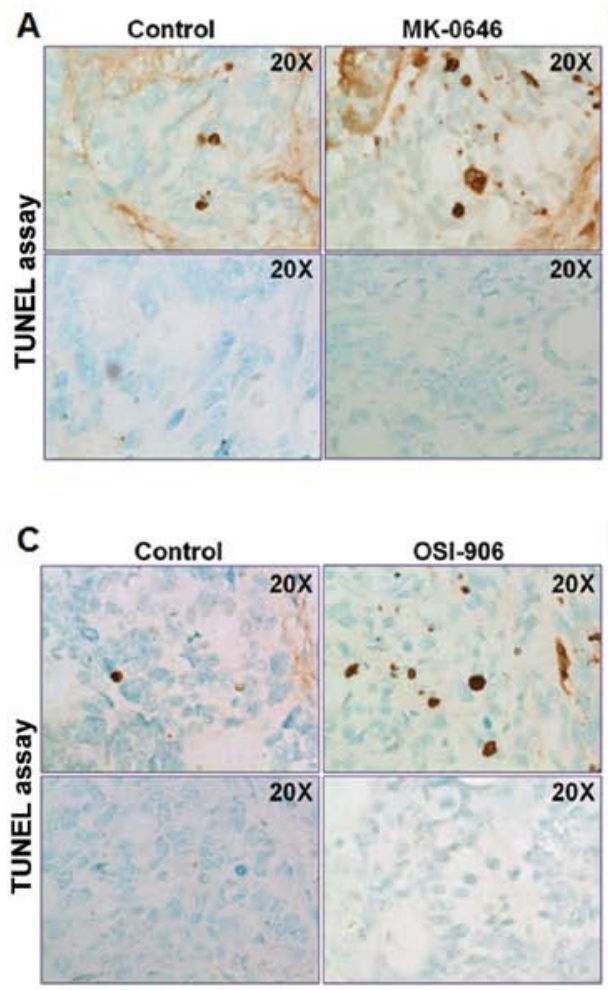

B

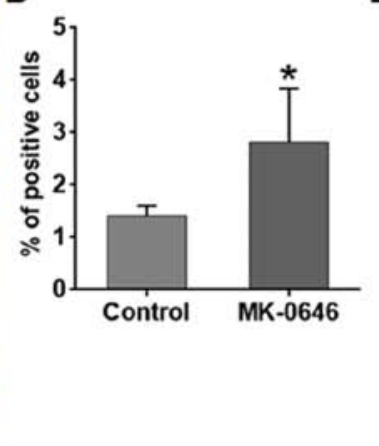

D

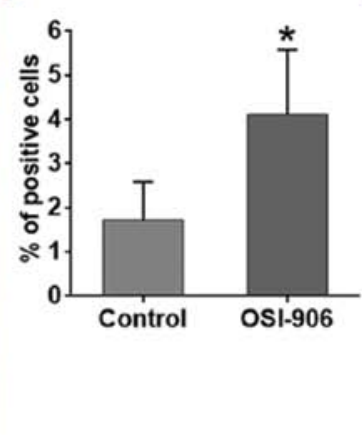

E
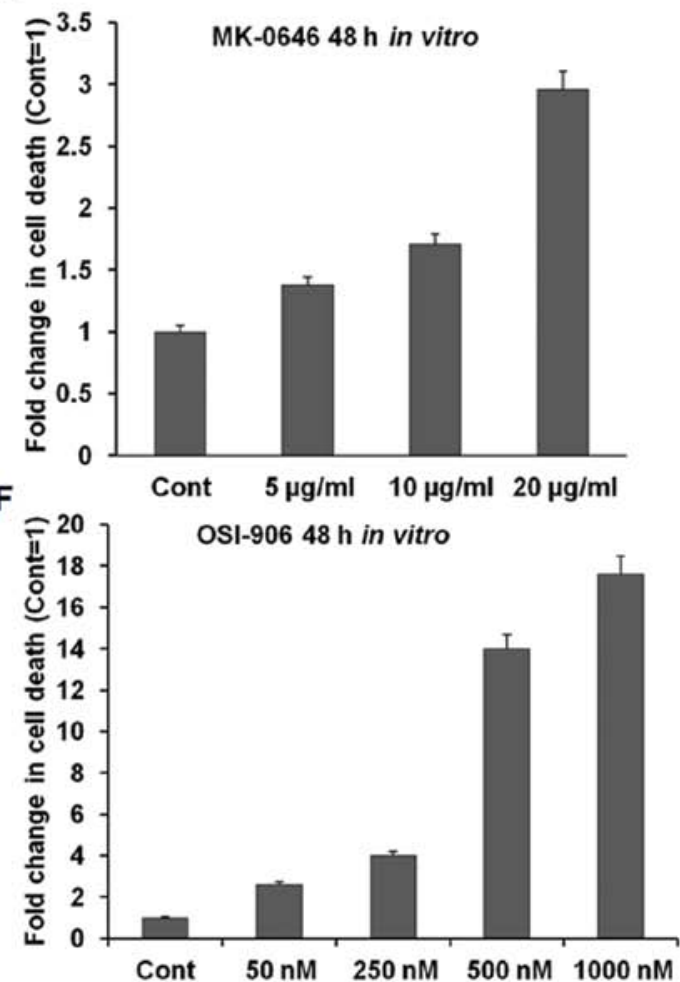

Figure 3. IGF-1R inhibition on apoptosis in vivo and in vitro. TUNEL assay was performed on both MK-0646 and OSI-906 treated and control xenograft paraffin-embedded slides and statistical analysis was determined (A, B, C and D). GEO cells were grown to 70-80\% confluency and treated with different doses of either MK-0646 or OSI-906 under GFDS for $48 \mathrm{~h}$ and performed DNA fragmentation assay as explained in Materials and methods (E and F).

volume and weight were significantly reduced in MK-0646 treated xenografts compared with the control (Fig. 1C and D). Results obtained by daily treatment of OSI-906 $(40 \mathrm{mg} / \mathrm{kg})$ for two weeks orally (Fig. 2A and B) were comparable to the MK-0646 treated tumor xenografts showing decrease in tumor growth. However, we observed $\sim 10 \%$ body weight reduction in OSI-906 treated mice compared with the MK-0646 treated mice (data not shown), which may be attributed to inhibition of insulin receptor by the dual kinase inhibitor.

IGF-1R inhibition of apoptosis in vivo and in vitro. We assessed the apoptosis level of control and drug-treated xenografts using TUNEL assays. Comparable results were obtained for both
IGF-1R antagonists. Both MK-0646 and OSI-906 treated GEO xenografts had statistically significant increase in apoptosis $(\mathrm{p}<0.05)$ as compared with the control tumors (Fig. 3A-D). Based on the pro-apoptotic effects of both MK-0646 and OSI-906 in vivo, DNA fragmentation was performed in vitro on GEO CRC cells to determine cell death following IGF-1R antagonist treatment (Fig. 3E and F). In accordance with the in vivo study, both MK-0646 and OSI-906 treatment showed significant increases in apoptosis demonstrating that IGF-1R inhibition elicits pro-apoptotic effects on CRC cells.

$I G F-1 R$ inhibition of cell proliferation in vivo and in vitro. Ki67 staining was performed to assess the proliferation level 

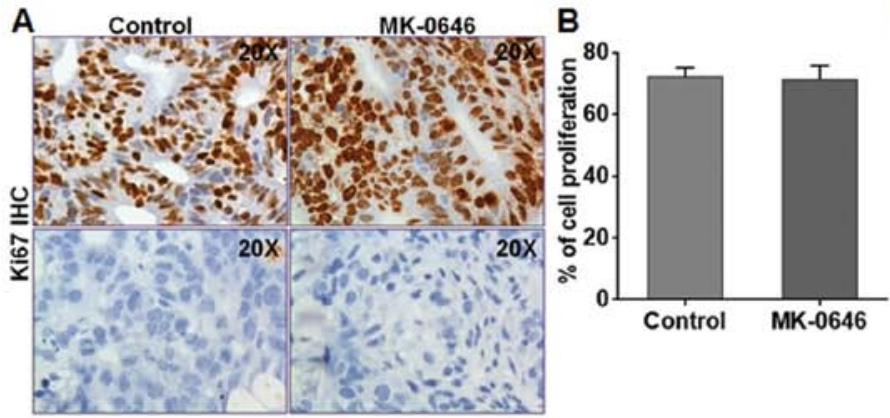

E
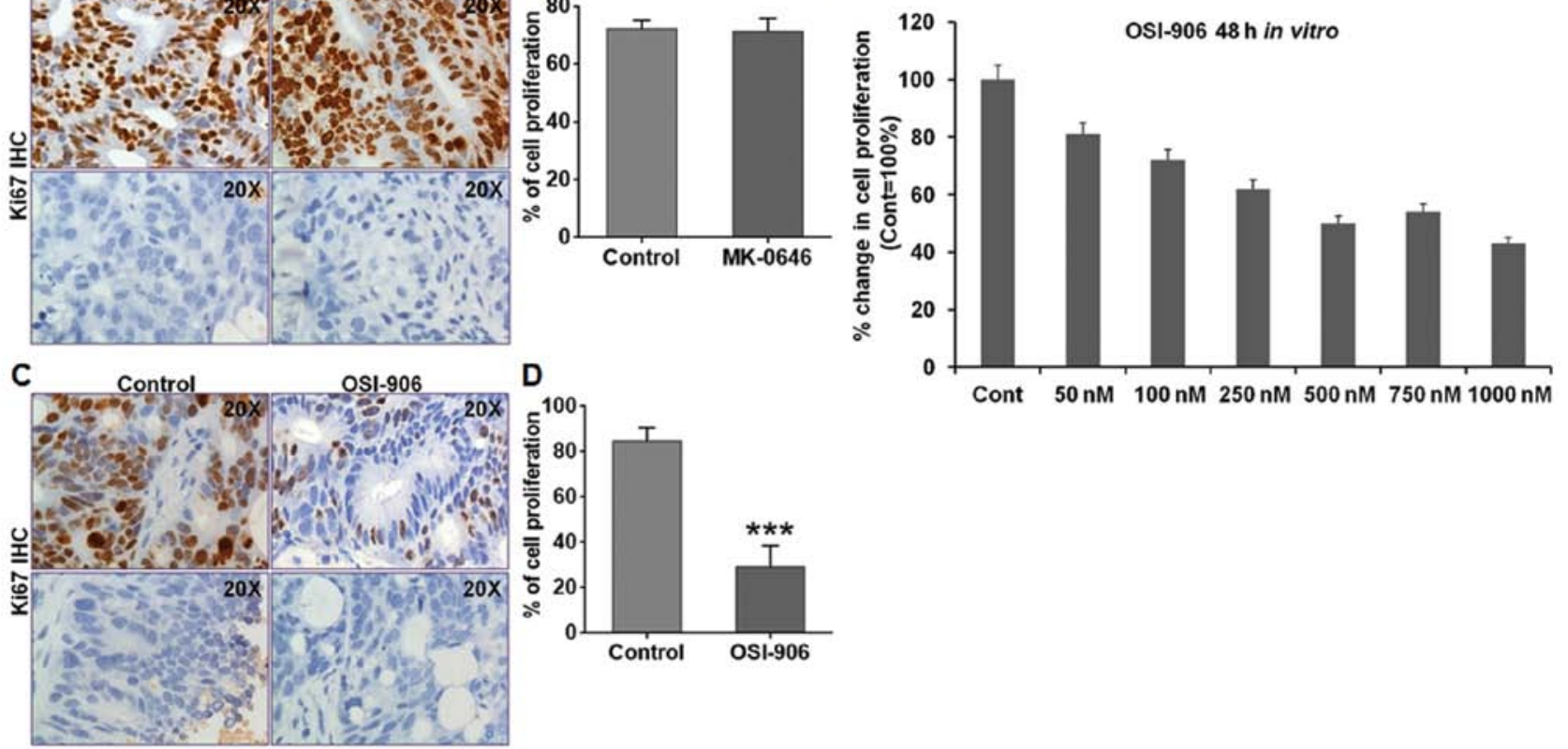

Figure 4. IGF-1R inhibition on cell proliferation in vivo and in vitro. Ki-67 staining was performed on both MK-0646 and OSI-906 treated and control xenograft paraffin-embedded slides and statistical analysis was determined (A, B, C and D). In order to determine the in vitro effects of IGF-1R inhibition on proliferation, MTT assay was performed with different doses of OSI-906 under GFDS for $48 \mathrm{~h}$ (E).

A
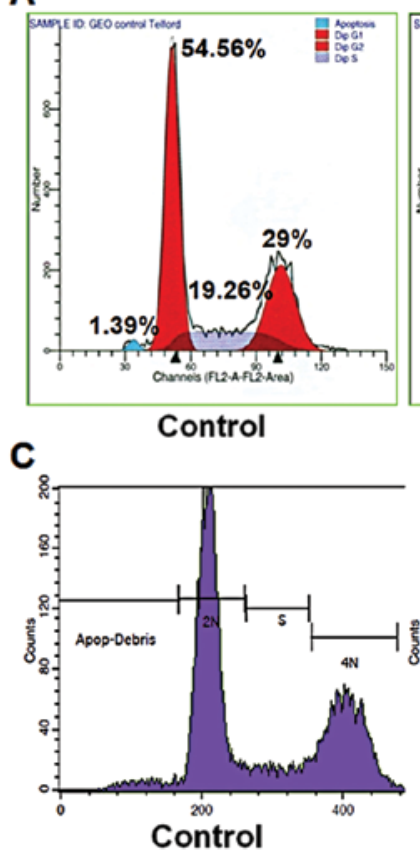

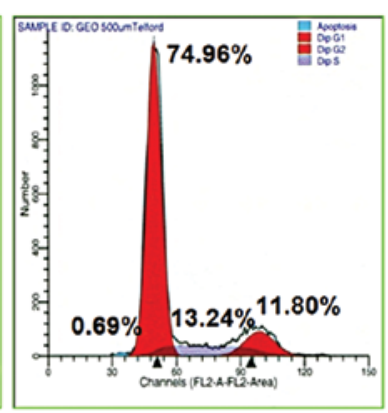

OSI-906 $0.5 \mu \mathrm{M}$

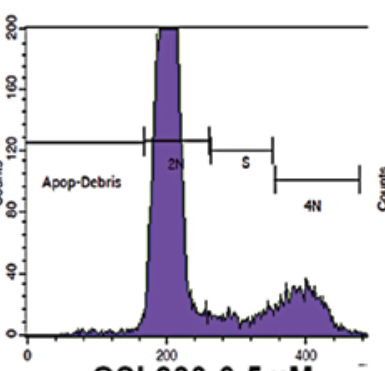

OSI-906 $0.5 \mu \mathrm{M}$
B

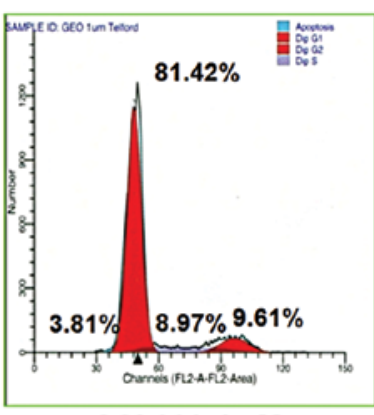

OSI-906 $1 \mu \mathrm{M}$

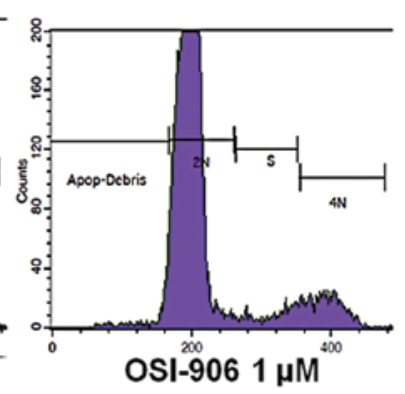

\begin{tabular}{|l|l|l|l|}
\hline & Cont & $0.5 \mu \mathrm{M}$ & $1 \mu \mathrm{M}$ \\
\hline $2 \mathrm{~N}$ & 34.74 & 53.39 & 54.44 \\
\hline $4 \mathrm{~N}$ & 20.12 & 9.59 & 6.26 \\
\hline
\end{tabular}

D

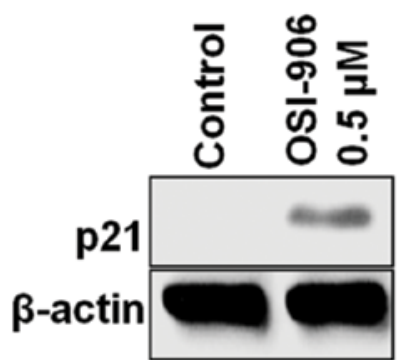

Figure 5. Effects of IGF-1R inhibition on cell cycle in vitro. GEO CRC cells were grown to 70-80\% confluence treated with OSI-906 under GFDS for $48 \mathrm{~h}$ and performed cell cycle analysis as explained in Materials and methods (A-C). Western blot analysis was performed on the OSI-906 treated GEO CRC cell lysates with p21 antibody using actin as a loading control (D).

on MK-0646 and OSI-906 treated GEO xenografts. MK-0646 treated GEO xenografts showed no change in the cell proliferation compared with the control (Fig. 4A and B). However, OSI-906 treated GEO xenografts showed a statistically significant reduction $(\mathrm{p}<0.05)$ in cell proliferation compared with the control (Fig. 4C and D). We also assessed cell proliferation of GEO CRC cells in vitro by MTT assay after treating with different concentrations of MK-0646 or OSI-906. OSI-906 treatment showed a decrease in cell proliferation (Fig. 4E), altered cell cycle in $\mathrm{G}_{0} / \mathrm{G}_{1}$ phase (Fig. 5A) and showed a decrease in the 4N DNA content (Fig. 5B and C). However, MK-0646 treatment showed no effect on cell cycle (data not shown). OSI-906 treatment also led to increase in p21 expression (Fig. 5D). 
A

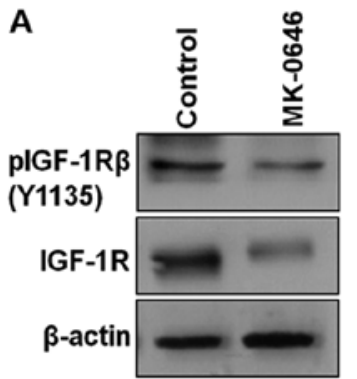

B

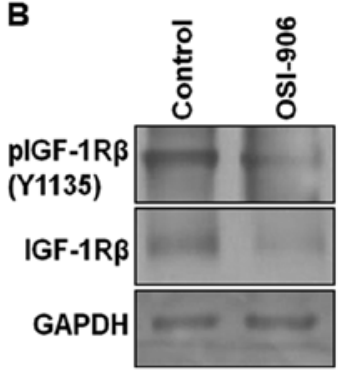

Figure 6. IGF-1R inhibition in vivo and in vitro decreases downstream substrates. GEO xenografts of both MK-0646 and OSI-906 were homogenized and western blot analyses were performed with antibodies to IGF-1R $\beta$ and pIGF-1R $\beta\left(\mathrm{Y}^{1135}\right)$. Actin and GAPGH were used as loading controls (A and $\mathrm{B})$.

$I G F-1 R$ treatment in vivo and in vitro decreases downstream substrates. Previous studies have shown that IGF-1R signaling exerts its anti-apoptotic effect through the IRS1/ IRS2/PI3K/Akt pathway (25-28). We determined the effects of MK-0646 and OSI-906 on the IGF-1R and its downstream signaling pathways on control and treated GEO CRC xenografts. The xenograft tumor samples were analyzed for the expression of molecules associated with the IGF-1R signaling pathway, including IGF-1R $\beta$ ( $\left.\mathrm{Y}^{1135}\right)$, Akt and pAkt $\left(\mathrm{S}^{473}\right)$. Inhibition of IGF-1R by MK-0646 and OSI-906 led to the downregulation of IGF-1R and its phosphorylation (Fig. 6A and $\mathrm{B}$ ), confirming the inhibitory effect of both antagonists on IGF-1R signaling. IGF-1R inhibition by MK-0646 or OSI-906

showed dephosphorylation of Akt at $\mathrm{S}^{473}$ site (data not shown). We next determined the effects of IGF-1R inhibition in vitro, using GEO cells following treatment with either MK-0646 or OSI-906. GEO cells were treated with MK-0646 or OSI-906 under GFDS conditions. Both MK-0646 and OSI-906 showed similar response on IGF-1R and p-IGF-1R in vitro (data not shown). Additionally, marked reduction in pAkt $\left(\mathrm{S}^{473}\right)$ was also observed in MK-0646 $(20 \mu \mathrm{g} / \mathrm{ml})$ or OSI-906 $(0.5 \mu \mathrm{M})$ treated cells (data not shown). These results showed that both antagonists MK-0646 and OSI-906 effectively inhibited IGF-1R and its downstream signaling.

IGF-1R inhibition downregulates the IAP molecule XIAP in vivo and in vitro. XIAP, an IAP (inhibitor of apoptosis) molecule and a key cell survival protein for inhibition of caspases, is a physiological substrate of Akt (29). Akt phosphorylates XIAP at $\mathrm{Ser}^{87}$ and regulates its autoubiquitination and degradation, and thereby stabilizes XIAP (29). Chowdhury et al have shown that XIAP is associated with pAKT and this association is disrupted following TGF $\beta$ treatment leading to XIAP degradation (30). Moreover, XIAP and survivin form a complex in the cytosol and this complex inhibits caspase activity as well as cell death and promotes tumor growth in vivo (31). Inhibition of the aberrant cell survival signaling of XIAP through destabilization of XIAP/survivin complexes leads to caspase reactivation and cell death $(30,32)$. We demonstrated that IGF-1R signaling pathway inhibition either by MK-0646 or OSI-906 both in vivo and in vitro downregulated Akt signaling, and this inhibition in turn leads to the downregulation of XIAP by immunohistochemical and western blot analysis (Fig. 7A-D). Next, we treated GEO
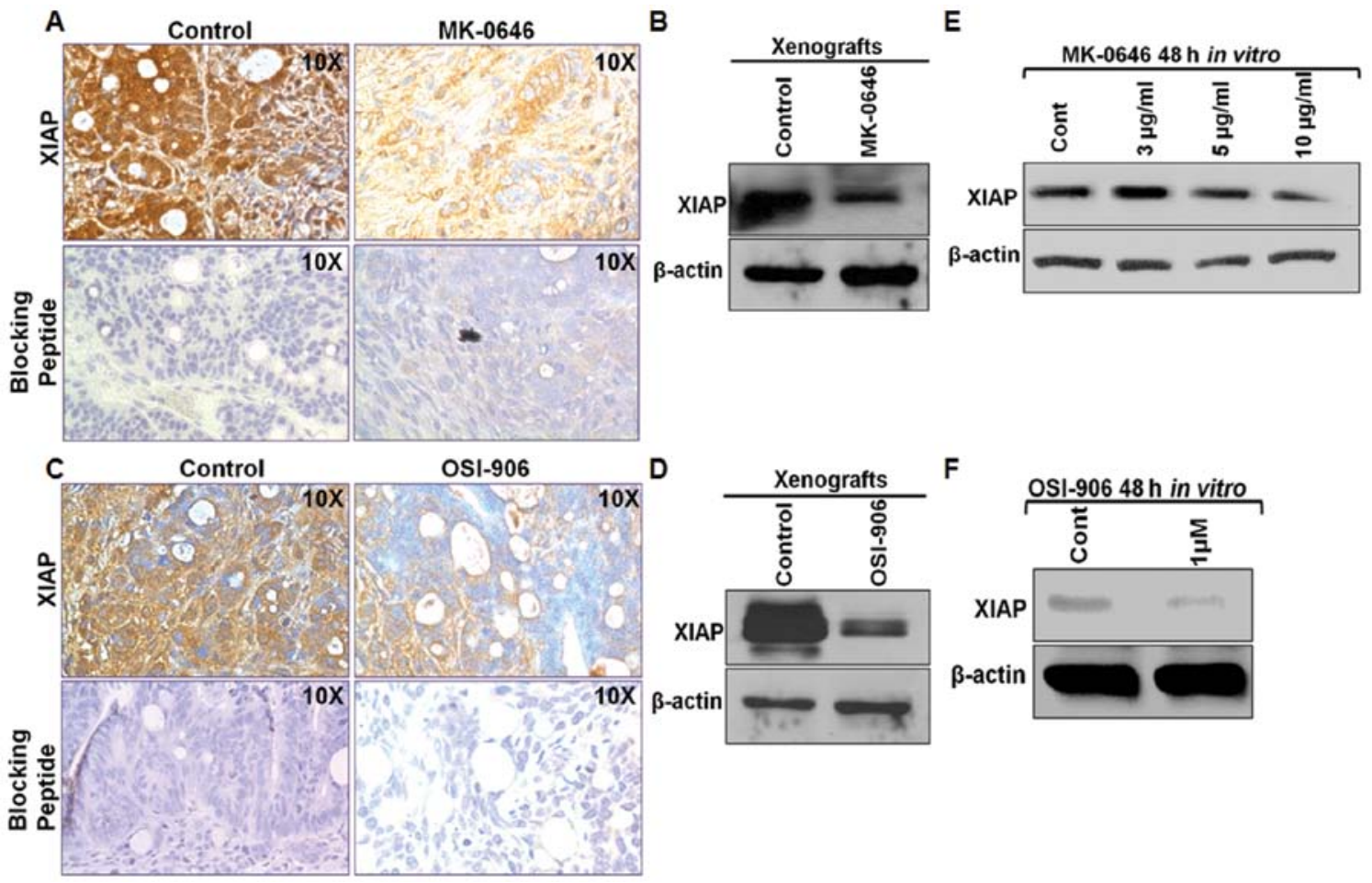

Figure 7. IGF-1R inhibition downregulates the IAP molecule XIAP in vivo and in vitro. Immunohistochemical analysis of XIAP protein was performed on both MK-0646 and OSI-906 treated paraffin-embedded xenografts. To confirm antibody specificity, a blocking peptide was used (A and B). Control and MK-0646 or OSI-906 treated GEO xenografts were analyzed by western blotting using anti-XIAP antibody (C and D). For in vitro analysis, GEO CRC cells were grown to 70-80\% confluence and treated with either MK-0646 or OSI-906 under GFDS for 48 h. Cells were harvested as described in Materials and methods and western blot analyses were performed with antibody to XIAP. Actin was used as a loading control (E and F). 
cells with the IGF-1R antagonist in vitro for $48 \mathrm{~h}$. Similar to the in vivo results, MK-0646 and OSI-906 both showed XIAP downregulation in the treated lysates compared to the control (Fig. 7E and F). These results showed that both antagonists exhibited their pro-apoptotic mechanism through inhibition of XIAP, an important downstream cell survival pathway molecule required for the survival of the IGF-1R-dependent CRC cells.

\section{Discussion}

Aberrant regulation of growth factors and their corresponding receptors play important roles in malignant progression (33-38). IGF-1R signaling pathway is prevalent in many cancers, including CRC (39-41). The IGF-IR gene has been reported to be overexpressed in human CRC (36) with $\sim 30-40 \%$ of all CRC being IGF-1R-dependent $(22,42)$. Therefore, IGF-1R signaling pathway is under intense investigation as an attractive candidate for the development of novel therapeutic strategies for anticancer treatment.

Buck et al inhibited the IGF-1R signaling pathway using OSI-906 in GEO xenograft tumors (18). It was reported that OSI-906 inhibited the growth of the GEO xenografts (18). Recently, we demonstrated that IGF-1R kinase inhibitor PQIP causes marked antitumor activity in these colon cancer celllines by abrogating the IGF-1R mediated activation of IRS1/Akt to inhibit survival signaling, and inducing apoptosis (10). In the present study, we analyzed the antitumor activity of a novel recombinant humanized monoclonal antibody, MK-0646 in CRC cells both in vivo and in vitro in comparison to OSI-906. Monoclonal antibodies against IGF-1R share a common mechanism of action, involving blockade of ligand-receptor interactions and decreased cell surface receptor through receptor internationalization and downregulation of the receptor (11,43-45). This leads to blockade of the PI3K/Akt signaling pathway (43-46). However, mechanisms associated with IGF-1R antagonist-mediated cell death are poorly understood. Our data demonstrated that MK-0646 decreased tumor growth in CRC xenografts in vivo and is supported by downregulation of IGF-1R and pIGF-1R $\beta$ in western blot analysis. MK-0646 demonstrated similar antitumor activity when compared to OSI-906.

IGF-1R signaling exerts its anti-apoptotic effect through IRS1/IRS2/PI3K/Akt pathway (10,22,25-28). We observed downregulation of IRS-1/2 and pAkt $\left(\mathrm{S}^{473}\right)$ and significant increase in tumor cell apoptosis after MK-0646 or OSI-906 treatment. Akt and its downstream molecular targets constitute a major cell survival pathway (47). XIAP, a prosurvival IAP, is a physiological substrate of Akt (29). Akt phosphorylates XIAP at $\mathrm{Ser}^{87}$ and reduces its degradation conferring resistance to caspase activation and apoptosis (29). Deregulation of IAP functions aberrantly prolonging cancer cell viability, and XIAP and survivin have been recognized for their role in tumor formation and are targets for cancer therapeutics (31). We made the novel observation that XIAP, a critical cell survival molecule that counteracts caspase activation and induction of apoptosis $(29,31)$ is downregulated with both MK-0646 and OSI-906 treatments demonstrating that XIAP is downstream of IGF-1R/Akt mediated control of aberrant cell survival responses. XIAP has been linked to cell survival and metastasis (48). XIAP/survivin complexes that mediate caspase inhibition have been shown to be a key cell survival mechanism for supporting the metastatic process (31). Previous studies in our laboratory have shown that destabilization of XIAP/survivin complexes by the TGF $\beta$ tumor suppressor signaling leads to inhibition of aberrant cell survival resulting in cell death $(30,32)$. Therefore, the IGF-1R signaling pathway and its downstream cell survival mediator XIAP may be potential dual targets for anticancer therapy.

In conclusion, this study demonstrated that MK-0646, a novel humanized IGF-1R monoclonal antibody, has comparable antitumor effects to IGF-1R small molecule inhibitor OSI-906, and may be a potential novel targeted therapy against IGF-1R-dependent subset of human CRC. Therefore, the results obtained in this study utilizing IGF-1R antagonists provide a rationale for further pre-clinical studies in order to dissect the IGF-1R signaling pathway to obtain full benefit from this receptor targeted therapy as a single agent or in combination against CRC as well as other solid tumors dependent upon IGF-1R signaling.

\section{Acknowledgements}

This work was supported by the NIH grants, CA 72001, CA 34432, CA 54807, CA 38173, to M.G.B.

\section{References}

1. Weber MM, Fottner C, Liu SB, Jung MC, Engelhardt D and Baretton GB: Overexpression of the insulin-like growth factor I receptor in human colon carcinomas. Cancer 95: 2086-2095, 2002.

2. Maloney EK, McLaughlin JL, Dagdigian NE, et al: An antiinsulin growth factor I receptor antibody that is a potent inhibitor of cancer cell proliferation. Cancer Res 63: 5073-5083, 2003.

3. Peters G, Gongoll S, Langner C, et al: IGF-1R, IGF-1 and IGF-2 expression as potential prognostic and predictive markers in colorectal-cancer. Virchows Arch 443: 139-145, 2003.

4. Ewing GP and Goff LW: The insulin-like growth factor signaling pathway as a target for treatment of colorectal carcinoma. Clin Colorectal Cancer 9: 219-223, 2010.

5. Asghar U, Hawkes E and Cunningham D: Predictive and prognostic biomarkers for targeted therapy in metastatic colorectal cancer. Clin Colorectal Cancer 9: 274-281, 2010.

6. McKinley ET, Bugaj JE, Zhao P, et al: 18FDG-PET predicts pharmacodynamic response to OSI-906, a dual IGF-1R/IR inhibitor, in preclinical mouse models of lung cancer. Clin Cancer Res 17: 3332-3340, 2011.

7. Goetsch L, Gonzalez A, Leger O, et al: A recombinant humanized anti-insulin-like growth factor receptor type I antibody (h7C10) enhances the antitumor activity of vinorelbine and anti-epidermal growth factor receptor therapy against human cancer xenografts. Int J Cancer 113: 316-328, 2005.

8. Allison AS, McIntyre MA, McArdle C and Habib FK: The insulin-like growth factor type 1 receptor and colorectal neoplasia: insights into invasion. Hum Pathol 38: 1590-1602, 2007.

9. Pollak MN, Schernhammer ES and Hankinson SE: Insulin-like growth factors and neoplasia. Nat Rev Cancer 4: 505-518, 2004.

10. Chowdhury S, Dominguez I, Sharratt E, Spernyak J, Brattain MG and Rajput A: Anti-tumor activity of IGF-1R kinase inhibitor PQIP in colon cancer. Clin Exp Pharmacol S4: 005, 2013.

11. Donovan EA and Kummar S: Role of insulin-like growth factor-1R system in colorectal carcinogenesis. Crit Rev Oncol Hematol 66: 91-98, 2008.

12. Atzori F, Traina TA, Ionta MT and Massidda B: Targeting insulin-like growth factor type 1 receptor in cancer therapy. Target Oncol 4: 255-266, 2009. 
13. Reidy-Lagunes DL, Vakiani E, Segal MF, et al: A phase 2 study of the insulin-like growth factor-1 receptor inhibitor MK-0646 in patients with metastatic, well-differentiated neuroendocrine tumors. Cancer 118: 4795-4800, 2012.

14. Reichert JM: Antibody-based therapeutics to watch in 2011 MAbs 3: 76-99, 2011.

15. King ER and Wong KK: Insulin-like growth factor: current concepts and new developments in cancer therapy. Recent Pat Anticancer Drug Discov 7: 14-30, 2012.

16. Heidegger I, Pircher A, Klocker H and Massoner P: Targeting the insulin-like growth factor network in cancer therapy. Cancer Biol Ther 11: 701-707, 2011.

17. Mulvihill MJ, Cooke A, Rosenfeld-Franklin M, et al: Discovery of OSI-906: a selective and orally efficacious dual inhibitor of the IGF-1 receptor and insulin receptor. Future Med Chem 1: 1153-1171, 2009.

18. Buck E, Gokhale PC, Koujak S, et al: Compensatory insulin receptor (IR) activation on inhibition of insulin-like growth factor-1 receptor (IGF-1R): rationale for cotargeting IGF-1R and IR in cancer. Mol Cancer Ther 9: 2652-2664, 2010.

19. Brattain MG,Levine AE, Chakrabarty S, Yeoman LC, Willson JK and Long B: Heterogeneity of human colon carcinoma. Cancer Metastasis Rev 3: 177-191, 1984.

20. Boyd DD, Levine AE, Brattain DE, McKnight MK and Brattain MG: Comparison of growth requirements of two human intratumoral colon carcinoma cell lines in monolayer and soft agarose. Cancer Res 48: 2469-2474, 1988.

21. Wang J, Yang L, Yang J, et al: Transforming growth factor beta induces apoptosis through repressing the phosphoinositide 3-kinase/AKT/survivin pathway in colon cancer cells. Cance Res 68: 3152-3160, 2008.

22. Hu YP, Patil SB, Panasiewicz M, et al: Heterogeneity of receptor function in colon carcinoma cells determined by cross-talk between type I insulin-like growth factor receptor and epidermal growth factor receptor. Cancer Res 68: 8004-8013, 2008.

23. Vanamala J, Reddivari L, Radhakrishnan S and Tarver C: Resveratrol suppresses IGF-1 induced human colon cancer cell proliferation and elevates apoptosis via suppression of IGF-1R/ Wnt and activation of p53 signaling pathways. BMC Cancer 10: $238,2010$.

24. Nakane PK: Recent progress in the peroxidase-labeled antibody method. Ann NY Acad Sci 254: 203-211, 1975.

25. Peruzzi F, Prisco M, Dews M, et al: Multiple signaling pathways of the insulin-like growth factor 1 receptor in protection from apoptosis. Mol Cell Biol 19: 7203-7215, 1999.

26. Ouban A, Muraca P, Yeatman T and Coppola D: Expression and distribution of insulin-like growth factor-1 receptor in human carcinomas. Hum Pathol 34: 803-808, 2003.

27. Myers MG Jr, Grammer TC, Wang LM, et al: Insulin receptor substrate-1 mediates phosphatidylinositol 3'-kinase and p70S6k signaling during insulin, insulin-like growth factor-1, and interleukin-4 stimulation. J Biol Chem 269: 28783-28789, 1994.

28. Kennedy SG, Wagner AJ, Conzen SD, et al: The PI 3-kinase/Akt signaling pathway delivers an anti-apoptotic signal. Genes Dev 11: 701-713, 1997.

29. Dan HC, Sun M, Kaneko S, et al: Akt phosphorylation and stabilization of X-linked inhibitor of apoptosis protein (XIAP). J Biol Chem 279: 5405-5412, 2004

30. Chowdhury S, Howell GM, Rajput A, et al: Identification of a novel TGFbeta/PKA signaling transduceome in mediating control of cell survival and metastasis in colon cancer. PLoS One 6: e19335, 2011.
31. Dohi T, Xia F and Altieri DC: Compartmentalized phosphorylation of IAP by protein kinase A regulates cytoprotection. Mol Cell 27: 17-28, 2007.

32. Chowdhury S, Howell GM, Teggart CA, et al: Histone deacetylase inhibitor belinostat represses survivin expression through reactivation of transforming growth factor beta (TGFbeta) receptor II leading to cancer cell death. J Biol Chem 286: 30937-30948, 2011.

33. Watson DS, Brotherick I, Shenton BK, Wilson RG and Campbell FC: Growth dysregulation and p53 accumulation in human primary colorectal cancer. Br J Cancer 80: 1062-1068, 1999.

34. Gryfe R, Swallow C, Bapat B, Redston M, Gallinger S and Couture J: Molecular biology of colorectal cancer. Curr Probl Cancer 21: 233-300, 1997.

35. Moschos SJ and Mantzoros CS: The role of the IGF system in cancer: from basic to clinical studies and clinical applications. Oncology 63: 317-332, 2002.

36. Sulkowski S, Kanczuga-Koda L, Koda M, Wincewicz A and Sulkowska M: Insulin-like growth factor-I receptor correlates with connexin 26 and Bcl-xL expression in human colorectal cancer. Ann NY Acad Sci 1090: 265-275, 2006.

37. Wu KD, Zhou L, Burtrum D, Ludwig DL and Moore MA: Antibody targeting of the insulin-like growth factor I receptor enhances the anti-tumor response of multiple myeloma to chemotherapy through inhibition of tumor proliferation and angiogenesis. Cancer Immunol Immunother 56: 343-357, 2007.

38. Hakam A, Yeatman TJ, Lu L, et al: Expression of insulin-like growth factor-1 receptor in human colorectal cancer. Hum Pathol 30: 1128-1133, 1999.

39. Reinmuth N, Liu W, Fan F, et al: Blockade of insulin-like growth factor I receptor function inhibits growth and angiogenesis of colon cancer. Clin Cancer Res 8: 3259-3269, 2002.

40. Sachdev D and Yee D: The IGF system and breast cancer. Endocr Relat Cancer 8: 197-209, 2001.

41. Surmacz E: Function of the IGF-I receptor in breast cancer. J Mammary Gland Biol Neoplasia 5: 95-105, 2000.

42. Buck E, Eyzaguirre A, Rosenfeld-Franklin M, et al: Feedback mechanisms promote cooperativity for small molecule inhibitors of epidermal and insulin-like growth factor receptors. Cancer Res 68: 8322-8332, 2008.

43. Gualberto A and Karp DD: Development of the monoclonal antibody figitumumab, targeting the insulin-like growth factor-1 receptor, for the treatment of patients with non-small-cell lung cancer. Clin Lung Cancer 10: 273-280, 2009.

44. Shang Y, Mao Y, Batson J, et al: Antixenograft tumor activity of a humanized anti-insulin-like growth factor-I receptor monoclonal antibody is associated with decreased AKT activation and glucose uptake. Mol Cancer Ther 7: 2599-2608, 2008.

45. Zha J and Lackner MR: Targeting the insulin-like growth factor receptor-1R pathway for cancer therapy. Clin Cancer Res 16: 2512-2517, 2010.

46. Chitnis MM, Yuen JS, Protheroe AS, Pollak M and Macaulay VM: The type 1 insulin-like growth factor receptor pathway. Clin Cancer Res 14: 6364-6370, 2008.

47. Agarwal E, Brattain MG and Chowdhury S: Cell survival and metastasis regulation by Akt signaling in colorectal cancer. Cell Signal 25: 1711-1719, 2013.

48. Mehrotra S, Languino LR, Raskett CM, Mercurio AM, Dohi T and Altieri DC: IAP regulation of metastasis. Cancer Cell 17: 53-64, 2010. 